\title{
Optical redox imaging indices discriminate human breast cancer from normal tissues
}

He N. Xu

Julia Tchou

Min Feng

Huaqing Zhao

Lin Z. Li 


\title{
Optical redox imaging indices discriminate human breast cancer from normal tissues
}

\author{
He N. Xu, ${ }^{a, b, *}$ Julia Tchou, ${ }^{c, d, e}$ Min Feng, ${ }^{a, b}$ Huaqing Zhao, ${ }^{f}$ and Lin Z. Li ${ }^{a, b, d, *}$ \\ aUniversity of Pennsylvania, Perelman School of Medicine, Department of Radiology, Molecular Imaging Laboratory, B6 Blockley Hall, \\ 423 Guardian Drive, Philadelphia, Pennsylvania 19104, United States \\ bUniversity of Pennsylvania, Perelman School of Medicine, Department of Biochemistry and Biophysics, Johnson Research Foundation, \\ Britton Chance Laboratory of Redox Imaging, R171 John Morgan Building, 3620 Hamilton Walk, Philadelphia, Pennsylvania 19104, United States \\ 'University of Pennsylvania, Perelman School of Medicine, Division of Endocrine and Oncologic Surgery, Department of Surgery, \\ West Pavilion 3rd Floor, 3400 Civic Center Boulevard, Philadelphia, Pennsylvania 19104, United States \\ dUniversity of Pennsylvania, Perelman School of Medicine, Abramson Cancer Center, 3400 Spruce Street, Philadelphia, Pennsylvania 19104, \\ United States \\ eUniversity of Pennsylvania, Perelman School of Medicine, Rena Rowan Breast Center, West Pavilion, 3rd Floor, 3400 Civic Center Boulevard, \\ Philadelphia, Pennsylvania 19104, United States \\ 'Temple University, School of Medicine, Department of Clinical Sciences, Kresge Room 218, 3440 North Broad Street, Philadelphia, \\ Pennsylvania 19140, United States
}

\begin{abstract}
Our long-term goal was to investigate the potential of incorporating redox imaging technique as a breast cancer $(\mathrm{BC})$ diagnosis component to increase the positive predictive value of suspicious imaging finding and to reduce unnecessary biopsies and overdiagnosis. We previously found that precancer and cancer tissues in animal models displayed abnormal mitochondrial redox state. We also revealed abnormal mitochondrial redox state in cancerous specimens from three BC patients. Here, we extend our study to include biopsies of 16 patients. Tissue aliquots were collected from both apparently normal and cancerous tissues from the affected cancer-bearing breasts shortly after surgical resection. All specimens were snap-frozen and scanned with the Chance redox scanner, i.e., the three-dimensional cryogenic NADH/Fp (reduced nicotinamide adenine dinucleotide/oxidized flavoproteins) fluorescence imager. We found both Fp and NADH in the cancerous tissues roughly tripled that in the normal tissues $(p<0.05)$. The redox ratio $\mathrm{Fp} /(\mathrm{NADH}+\mathrm{Fp})$ was $\sim 27 \%$ higher in the cancerous tissues $(p<0.05)$. Additionally, $\mathrm{Fp}$, or $\mathrm{NADH}$, or the redox ratio alone could predict cancer with reasonable sensitivity and specificity. Our findings suggest that the optical redox imaging technique can provide parameters independent of clinical factors for discriminating cancer from noncancer breast tissues in human patients. ๑ 2016 Society of Photo-Optical Instrumentation Engineers (SPIE) [DOI: 10.1117/1.JBO.21.11.114003]
\end{abstract}

Keywords: mitochondria; NADH; flavoproteins containing flavin adenine dinucleotide; redox ratio; clinical biopsy.

Paper 160236SSRR received Apr. 12, 2016; accepted for publication Oct. 27, 2016; published online Nov. $29,2016$.

\section{Introduction}

There is a clinical need to develop complementary or alternative methods that are objective, unambiguous, rapid, and costeffective for early detection and diagnosis of breast cancer (BC). The current gold standard to establish BC diagnosis is histological examination of a biopsy. However, this method only provides tissue information after biopsy procedure and is at a disadvantage of subjective interpretation of morphological features and can also be time-consuming since it involves postprocessing of specimens. Although BC screening using mammography has been shown to reduce overall death by about $20 \%$, it does harm to patients with a high rate of false-positivity and overdiagnosis. ${ }^{1-3}$ A high percentage $(>50 \%)$ of patients have a biopsy that yields a benign result. This indicates that over half of the women who undergo a biopsy experience an unnecessary invasive procedure. ${ }^{3,4}$ Thus, there is an unmet need for developing technologies to improve the diagnosis of BC.

Since cancer cells exhibit altered metabolism, molecular and metabolic imaging methods could generate information that

*Address all correspondence to: Lin Z. Li, E-mail: linli@mail.med.upenn.edu; He N. Xu, E-mail: hexu3897@yahoo.com relates to cell metabolism and biochemistry, thus providing pathological information. In addition, altered metabolism could occur prior to the morphological changes and may exhibit metabolic heterogeneity at a higher than normal level. Such information could have diagnostic and prognostic value complementary to conventional pathological tools. The present work is part of our series of investigations aiming to identify diagnostic and prognostic cancer biomarkers based on molecular imaging of the mitochondrial redox state..$^{5-15}$

Redox balance is one of the basic needs supported by altered cellular metabolism in dividing cells. ${ }^{16}$ The cellular redox state mediates many biological activities, such as metabolism, differentiation, proliferation, survival, apoptosis, invasion, migration, reactive oxygen species generation, gene expression, and signaling. ${ }^{16-20}$ Among many of the redox systems, nicotinamide adenine dinucleotide (NAD) and NAD-coupled redox regulation have been recognized to play important roles in cancer biology..$^{20,21}$ The mitochondrial redox state in tissue can be optically determined by measuring the endogenous fluorescence from mitochondrial NADH and oxidized flavoproteins (Fp) containing flavin adenine dinucleotide (FAD) as pioneered by

$1083-3668 / 2016 / \$ 25.00$ @) 2016 SPIE 
Chance and coworkers. ${ }^{22-26} \mathrm{NADH}$ and $\mathrm{FADH}_{2}$ serve as electron carriers during the adenosine triphosphate-producing catabolic processes. The optical redox ratios [e.g., Fp/NADH or the normalized $\mathrm{Fp}$ redox ratio $=\mathrm{Fp} /(\mathrm{NADH}+\mathrm{Fp})]$ are sensitive to the mitochondrial NAD-coupled redox state and correlate with the $\mathrm{NAD}^{+} / \mathrm{NADH}$ redox potentials determined by both biochemical assays and liquid chromatography-mass spectrometry. ${ }^{25,27-29}$

Although many fluorescence spectroscopic studies of clinical breast samples have reported higher than normal NADH and/or $\mathrm{Fp}$ in cancer tissues, ${ }^{30-38}$ fluorescence imaging of NADH, Fp, and the redox ratios provides spatial and metabolic heterogeneity of tissue, ${ }^{5,38}$ which might provide additional valuable information for revealing disease state and discriminating between premalignant and normal tissues. ${ }^{12,39,40}$ In recent years, more reports on imaging NADH and Fp fluorescence and the redox ratio of clinical tissue samples have become available. For example, multiphoton microscopy has been utilized to explore the feasibility of distinguishing head and neck cancer tissues from the normal ones ${ }^{41}$ and differentiating normal low-risk human ovaries from normal high-risk and cancerous ovaries. ${ }^{42}$ However, few studies have investigated the feasibility of redox imaging of the clinical biopsy specimens of BC.

Employing the Chance redox scanner, ${ }^{22,24}$ a three-dimensional cryogenic fluorescence imager of NADH, Fp, and the redox ratios at a resolution as low as $50 \times 50 \times 20 \mu \mathrm{m}^{3}$, we have found that precancer and cancer tissues in the animal models displayed altered mitochondrial redox state and high level of redox heterogeneity. ${ }^{5}$ Particularly, our findings from imaging the core biopsies from three BC patients showed that Fp signal can be up to 10-fold higher in cancerous tissues than adjacent normal tissues. ${ }^{10,11}$ In the present study, we report our investigation on the quantification of the mitochondrial redox state and its heterogeneity of biopsies from $16 \mathrm{BC}$ patients and the redox difference between the cancerous tissues and their normal counterparts. Part of the data presented in this report has been published in an SPIE conference proceedings paper. ${ }^{43}$

\section{Materials and Methods}

\subsection{Tissue Specimen Collection}

Tissue collection from 16 female patients was performed during $\mathrm{BC}$ surgeries according to a protocol approved by the internal review board of the University of Pennsylvania. Tissue aliquots were collected by the surgeon at room temperature from both cancerous and adjacent normal-appearing (normal) tissues from the resected breasts/tissue chunks after their sitting in the surgical tray for a period of $25 \pm 19 \mathrm{~min}$. The tissue aliquots were snap-frozen with liquid nitrogen (within several minutes, mostly within $1 \mathrm{~min}$ ). The tumor and normal tissues were identified by palpation and further evaluated by a licensed pathologist. As normal tissue is primarily fat and tumor induces a dense fibrotic stromal reaction, which is firmer and denser than normal tissue, the surgeon and pathologist can discern normal from tumor easily by palpation and by inspecting appearance of the tissue. The described procedure is standard tissue banking protocol and quality assurance is routinely performed to confirm tumor versus normal tissue histology. For our study, due to tissue sample size being very small (needle biopsy, $\sim 2 \mathrm{~mm}$ in diameter, and some of them being exhausted while subjected to redox scanning or fixation for hematoxylin and eosin staining), histological staining on the remaining tissue became infeasible
Table 1 The clinical characteristics of the 16 BC patients.

\begin{tabular}{|c|c|c|c|c|c|}
\hline Patient & Type & Age & $\begin{array}{c}\text { Tumor } \\
\text { size }(\mathrm{cm})\end{array}$ & Grade & $\begin{array}{l}\text { Nodal } \\
\text { status }\end{array}$ \\
\hline 1 & TNBC & 40 & 2.3 & 3 & $\mathrm{~N}$ \\
\hline 2 & TNBC & 41 & 3.2 & 3 & $\mathrm{~N}$ \\
\hline 3 & $\mathrm{ER}^{+}$ & 83 & 5.5 & n.d. & $\mathrm{P}$ \\
\hline 5 & TNBC & 50 & 8 & 3 & $\mathrm{~N}$ \\
\hline 6 & $\mathrm{ER}^{+}$ & 39 & 5 & 2 & $\mathrm{P}$ \\
\hline 7 & $\mathrm{ER}^{+}$ & 45 & 1.8 & 1 & $\mathrm{P}$ \\
\hline 8 & $\mathrm{ER}^{+}$ & 55 & 0.6 & 1 & n.d. \\
\hline 9 & $\mathrm{ER}^{+}$ & 34 & 2.8 & n.a. & $\mathrm{P}$ \\
\hline 10 & TNBC & 65 & 2.7 & 3 & $\mathrm{P}$ \\
\hline 11 & $\mathrm{ER}^{+}$ & 44 & $3.2,1.5,2.0$ & 2 & $\mathrm{P}$ \\
\hline 12 & $\mathrm{ER}^{+}$ & 49 & 9.5 & n.a. & $\mathrm{N}$ \\
\hline 13 & $\mathrm{ER}^{+} / \mathrm{Her}^{+}$ & 56 & $2.5,1.5$ & 2 & $\mathrm{P}$ \\
\hline 14 & TNBC & 55 & 2.5 & 3 & $\mathrm{~N}$ \\
\hline 15 & $\mathrm{ER}^{+}$ & 49 & 3 & 2 & $\mathrm{~N}$ \\
\hline 16 & $\mathrm{ER}^{+}$ & 48 & 0.9 & 1 & $\mathrm{P}$ \\
\hline 17 & $\mathrm{ER}^{+} / \mathrm{Her}^{+}$ & 23 & 5 & n.a. & $\mathrm{N}$ \\
\hline
\end{tabular}

Note 1: Biopsies from patient 4 were lost during tissue handling. $\mathrm{N}$, negative; $\mathrm{P}$, positive; n.a., not available; n.d., not done.

for some samples. Therefore, we relied on visual inspection and palpation as described previously to identify tumor and normal tissues.

Table 1 lists the relevant clinical information on the 16 female patients with 5 triple negative breast cancer (TNBC), 9 estrogen receptor positive $\left(\mathrm{ER}^{+}\right)$, and $2 \mathrm{ER}^{+} /$human epidermal growth factor receptor 2 positive $\left(\mathrm{Her} 2^{+}\right)$. With the exception that a few specimens (one tumor and three normal tissue specimens) were obtained as tissue blocks $(\sim 1$ to $3 \mathrm{~cm}^{2}$ and $\sim 1$ - to 2 -cm thick), all other tissue samples were collected as core needle biopsies $\left(\sim 2 \mathrm{~mm}^{2} \times 10-\right.$ to $20-\mathrm{mm}$ long) obtained from the resected breast tissue chunks using a 12-gauge biopsy needle. All snap-frozen tissue aliquots were stored in liquid nitrogen at all times before redox scanning. More details on specimen collection procedures can be found elsewhere. ${ }^{10,11}$

\subsection{Tissue Embedding and Redox Scanning}

The tissue embedding procedure used in this study has been described in detail elsewhere. ${ }^{6,44,45}$ Briefly, the frozen biopsies were embedded with a chilled special mounting media $\left(\mathrm{H}_{2} \mathrm{O}\right.$ : ethanol: glycerol $=60: 10: 30$, freezing point $\left.-30^{\circ} \mathrm{C}\right)$. To facilitate day-to-day signal comparison, reference standards of frozen NADH and FAD solutions of known concentrations were placed adjacent to the biopsies. The concentrations of NADH and FAD stock solutions were determined by their 
optical absorptions at 340 and $450 \mathrm{~nm}$, respectively, using a UV/ VIS spectrometer. The stock solutions were further diluted to the desired concentrations for preparing the reference standards. The linear dynamic range of the instrument was determined when deemed necessary by imaging and quantifying the fluorescence of frozen NADH (and FAD) solutions of known concentrations as detailed elsewhere. ${ }^{44,45}$ The concentrations of the reference standards were chosen within the linear dynamic range.

The Chance redox scanner is equipped with a miller that mills the sample surface flat and exposes the tissue sample for desired depth. There are two filter wheels that spin at $60 \mathrm{~Hz}$ synchronously for four time-sharing fluorescence channels at the chosen excitation and emission wavelengths. The excitation lights from a mercury/metal halide lamp are directed onto the sample surface through the central optical fiber in a raster-scanning light guide, which also collects the emission light from the sample via six peripheral fibers built in the guide. The image resolution is limited by the diameter of central optical fiber ranging 50 to $200 \mu \mathrm{m}$ (50 and $100 \mu \mathrm{m}$ in the present study). A photomultiplier tube (Hamamatsu R928) is used as the detector. The instrument is automated and controlled by a PC.

For this study, the embedded tissue samples were repeatedly grounded by the miller to expose surface planes at various depths and scanned under liquid nitrogen by the Chance redox scanner. The optical filters for NADH and Fp channels were: $365 \pm 13 \mathrm{~nm}(\mathrm{Ex}) / 455 \pm 35 \mathrm{~nm}(\mathrm{Em})$ and $440 \pm 10 \mathrm{~nm}(\mathrm{Ex}) /$ $515 \pm 15 \mathrm{~nm}(\mathrm{Em})$, respectively. The pixel size is 50 to $200 \mu \mathrm{m}$. Two to four planes spacing 100 to $200 \mu \mathrm{m}$ were scanned for each specimen. The scan depth of the first imaging plane is defined as $0 \mu \mathrm{m}$.

\subsection{Data Analysis}

The acquired images were processed using a customized MATLAB $^{\circledR}$ program. The nominal concentrations of both Fp and NADH in the tissues were obtained by comparing their fluorescence intensities with that of the corresponding frozen solution standards. These nominal concentrations were only used for comparison of results from different imaging sessions and may deviate significantly from the true NADH and Fp concentrations in the tissue. The redox indices, including NADH, Fp, and the redox ratios of each plane, were extracted from each imaging plane of the biopsies. The mean values of the redox indices of each imaging plane were further averaged across planes to obtain the global average values for each patient. These data were statistically analyzed with the Wilcoxon signed-ranks test for paired data and two sample $t$-test for unpaired data. The receiver operating characteristic (ROC) analysis was performed to obtain sensitivity and specificity for the differentiation between tumor and normal tissues $P<0.05$ is considered statistically significant.

\section{Results}

\subsection{Redox Indices Discriminate between Cancerous and Normal Tissues}

Figure 1 shows the typical redox images and the corresponding histograms of a representative pair of the normal and cancerous tissues from the same patient. By referencing the color bar or reading the numbers under the $x$-axis of the histograms, we can readily see that the cancer tissue has much higher Fp and NADH average values than the normal counterpart. The mean values and their standard deviations of the redox indices for each group are shown in Table 2. Both NADH and Fp signals in the cancerous tissues roughly tripled to quadrupled those in the normal tissues; and the normalized Fp redox ratio $\mathrm{Fp} /$ $(\mathrm{NADH}+\mathrm{Fp}$ ) was about $27 \%$ higher in the cancerous tissues than in the normal ones. Figure 2 delineates the box plots of these redox indices.

A Wilcoxon signed-rank test showed that between the normal and tumor groups, there is a significant difference in each of the redox indices as listed in Table 2. Except that NADH/Fp redox ratio is significantly higher in the normal group ( $p=0.017), \mathrm{Fp}$, $\mathrm{NADH}$, and the Fp redox ratio Fp/(NADH + Fp) are all significantly higher in the tumor group ( $p=0.001,0.003$, and 0.003 , respectively). Furthermore, all tumor specimens had a higher Fp than the normal tissue except for one patient (patient 17), who had a higher Fp value in her normal tissue [Fig. 3(a)]. In 13 out of 16 patients, we observed a higher NADH in the tumor tissue than in the normal tissue whereas three patients had a higher NADH value in their normal tissue [Fig. 3(b)]. As shown in Fig. 3(c), 13 patients had a higher Fp redox ratio $\mathrm{Fp} /(\mathrm{NADH}+\mathrm{Fp})$ in the tumor tissue than their normal tissue. In 12 out of 16 patients, the normal tissue had a higher $\mathrm{NADH} / \mathrm{Fp}$ than the tumor tissue whereas four patients had a higher $\mathrm{NADH} / \mathrm{Fp}$ value in the tumor tissue.

We further tested the discriminating power of the redox indices for cancer. The ROC curves for Fp, NADH, and the Fp redox ratio are delineated in Fig. 4. As shown in Table 3, Fp has the largest area under curve (AUC). Both Fp and NADH can discriminate cancer from normal tissues with $81 \%$ sensitivity and $69 \%$ specificity. For the Fp redox ratio Fp/(NADH + Fp), $75 \%$ sensitivity with $69 \%$ specificity can be achieved.

\subsection{Relationship between Tumor Redox Indices and Conventional Clinical Risk Factors}

We further investigated if tumor redox indices would relate to the molecular type or tumor grade. Student $t$-tests performed for the tumor group showed no significant difference in any redox index between $\mathrm{ER}^{+}$and TNBC or between TNBC and nonTNBC subgroups. A $t$-test performed for 12 out of 16 patients, who were given tumor grades, showed no significant difference in any redox index between the low grade group (1 and 2) and high grade group (3). A $t$-test performed for 15 out of 16 patients, who were given nodal status, showed no significant difference in any tumor redox index between node positive and node negative groups. Nor was there a significant correlation between tumor redox indices and tumor size. Among $9 \mathrm{ER}^{+}$ patients, neither could we find a correlation between tumor size and tumor redox indices.

\section{Discussion}

In comparison with our previous study of the breast tissue specimens of three BC patients, the present study includes biopsies from 16 patients and demonstrates both NADH and Fp contents are significantly higher in tumor than in normal tissue based on the global averaging method; whereas previously only Fp difference between cancer and noncancer was significant due to relatively small sample size. ${ }^{10,11}$ The globally averaged Fp redox ratio was found in the present work significantly higher in tumor than in normal tissue with a $p$ value of 0.003 whereas previously 


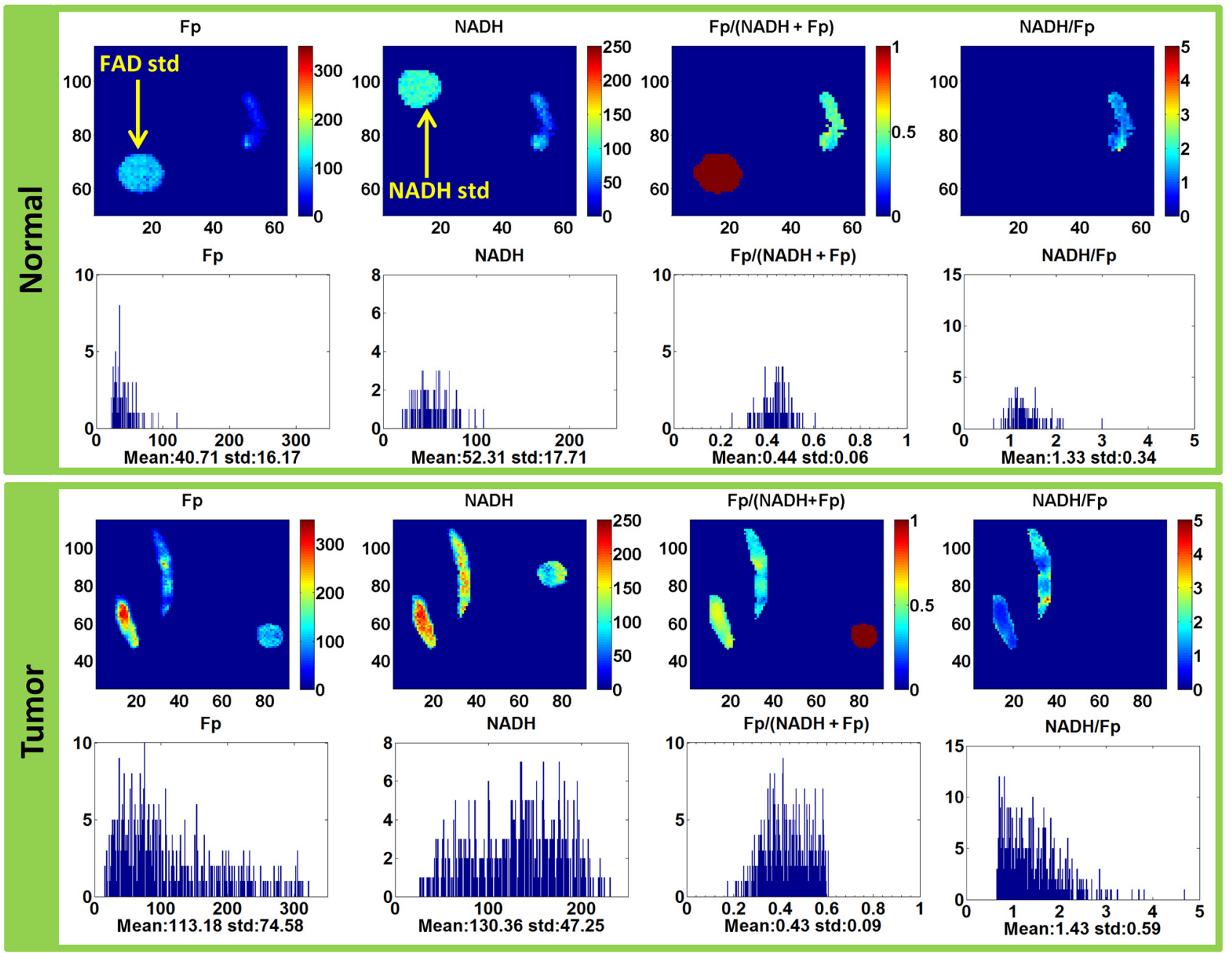

Fig. 1 The typical redox images and the corresponding histograms of a normal tissue biopsy (image plane depth $=600 \mu \mathrm{m}$ ) and a tumor tissue biopsy (image plane depth $=300 \mu \mathrm{m}$ ) of the same patient (image matrix $128 \times 128$, spatial resolution $100 \mu \mathrm{m}$ ). The Fp or NADH images show the nominal concentrations in $\mu \mathrm{M}$, the Fp redox ratio ranges between 0 and 1. The $x$-axes of the corresponding histograms represent the concentrations or redox ratios. The $y$-axes of the histograms represent the number of pixels in the image plane having a specific value of concentration or the redox ratio. The round spots in the images are corresponding FAD or NADH reference standards.

the $p$ value was only a borderline significance (0.07). Higher Fp redox ratio indicates that the tumor tissue was more oxidized than the normal tissue on average. In addition, we also found that Fp or NADH or the Fp redox ratio alone could be used as a discriminating index for $\mathrm{BC}$ with reasonable sensitivity and specificity (Table 3), although they should be further improved before they can be useful clinically. The nominal

Table 2 The redox indices of the normal and cancer groups $(N=16$ for each group, mean $\pm S D$ ).

\begin{tabular}{lcccc} 
& $\mathrm{Fp}(\mu \mathrm{M})$ & $\mathrm{NADH}(\mu \mathrm{M})$ & $\mathrm{Fp} /(\mathrm{NADH}+\mathrm{Fp})$ & $\mathrm{NADH} / \mathrm{Fp}$ \\
\hline Normal & $83 \pm 90$ & $84 \pm 54$ & $.44 \pm .11$ & $1.63 \pm .94$ \\
tumor & $308 \pm 320$ & $217 \pm 212$ & $.56 \pm .08$ & $.95 \pm .42$ \\
$p^{\mathrm{a}}$ & .001 & .003 & .003 & .017 \\
\hline
\end{tabular}

aWilcoxon signed-rank test. concentrations are used to indicate the intensities of NADH and Fp signals and to facilitate comparison of data obtained from different samples or on different days. These nominal concentrations may deviate largely from the true concentrations of these two species in tissue.

We observed that NADH nominal concentration in tumor tissue is 2.6-fold of that in normal tissue. This is consistent with what was reported in the literature. For example, a biochemical assay of breast tissues resulted in 1.9 times higher NADH in cancer tissue than in the normal surrounding tissue. ${ }^{33}$ In that study, NADH was extracted from both fresh tissues (processed within 3 days after tissue resection) and frozen tissue (maintained in $-20^{\circ} \mathrm{C}$ for 2 to 4 months). Some spectroscopic studies also showed that NADH intensity in the BC tissue was $\sim 2$ to 3 fold of the normal counterpart as estimated by the fluorescence intensity at $\sim 460 \mathrm{~nm},{ }^{34-36}$ although another spectroscopic study showed NADH in the BC tissue was only $\sim 15 \%$ higher than that in the normal ones. ${ }^{37}$ Studies looking at fixed tissue slides also found higher NADH fluorescence in the breast tumor region compared to the normal region. ${ }^{46,47}$ 

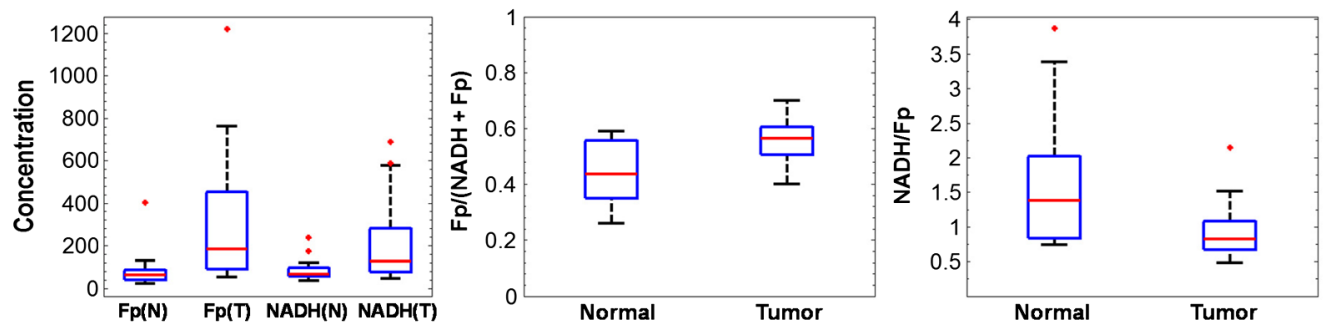

Fig. 2 Box plots of the redox indices representing the lower adjacent value, first quartile, median, third quartile, and upper adjacent value for the normal and tumor groups ( $N$, normal; $\mathrm{T}$, tumor). The red dots are outliers.
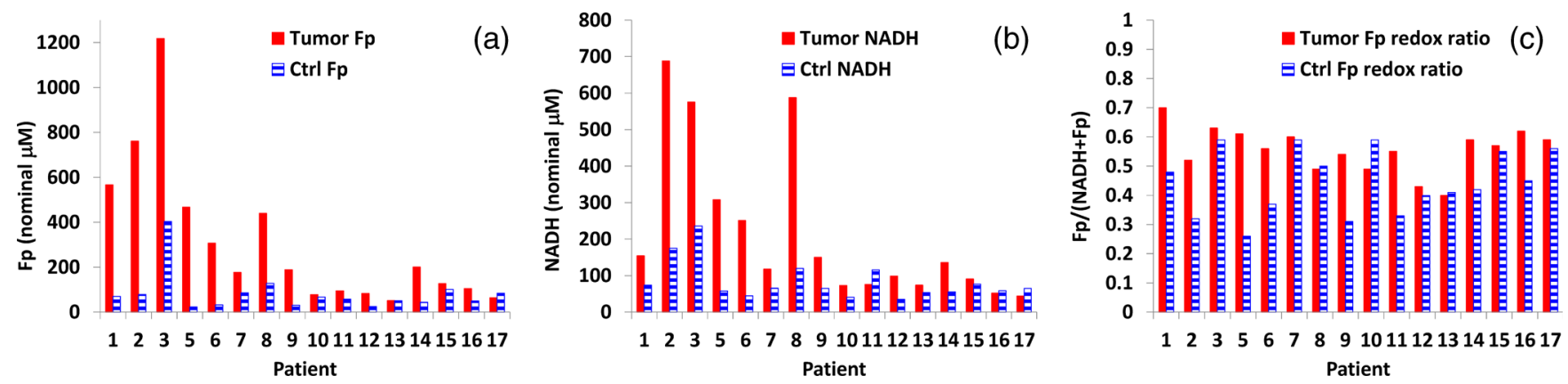

Fig. 3 The redox indices for paired tumor (bars in red) and normal (bars with blue patterns) tissues from individual patients: (a) Fp, (b) NADH, and (c) the Fp redox ratio.
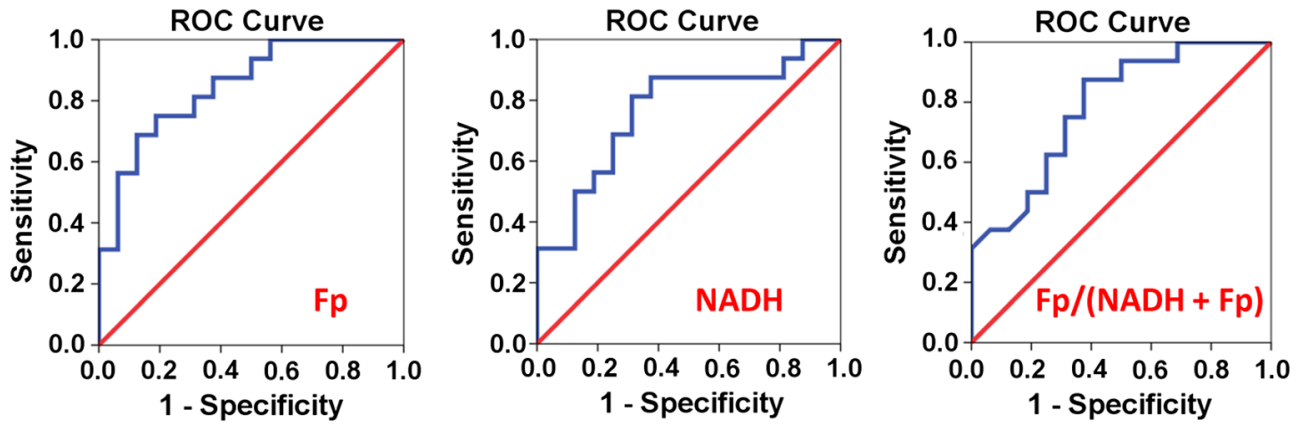

Fig. 4 The ROC curves for the redox indices, i.e., Fp, NADH, and the Fp redox ratio.

We observed Fp nominal concentration in breast tumor tissue is 3.7-fold of that in the normal tissue. We have not come across any reported Fp contents in tumor tissue as measured by a biochemical assay. Spectroscopic studies have shown higher fluorescence intensities in the range between 500- and 700-nm in breast tumor tissues than in normal ones ${ }^{30,31}$ and 500- to

Table 3 The ROC analysis of the redox indices.

\begin{tabular}{lccc} 
& $\mathrm{Fp}$ & $\mathrm{NADH}$ & $\mathrm{Fp} /(\mathrm{NADH}+\mathrm{Fp})$ \\
\hline AUC & 0.85 & 0.77 & 0.79 \\
$95 \% \mathrm{Cl}$ & $0.72,0.98$ & $0.60,0.94$ & $0.63,0.94$ \\
sensitivity, specificity & $81 \%, 69 \%$ & $81 \%, 69 \%$ & $75 \%, 69 \%$ \\
$p$ & .001 & .010 & .006 \\
\hline
\end{tabular}

600-nm autofluorescence was shown flavins origin. ${ }^{32}$ Some quantitative spectroscopic studies involving 28 to 34 patients reported $3.5-$ fold $^{35}$ and 2.5 -fold ${ }^{34}$ higher Fp (estimated from the fluorescence intensity at $\sim 525 \mathrm{~nm}$ ) in BC tissues compared to their normal counterparts.

We have not found any literature reporting the Fp redox ratio in clinical breast biopsy samples. Optical redox ratios of the clinical specimens for other forms of cancer have been reported. For example, it was shown that normal low-risk human ovaries were associated with a lower Fp redox ratio whereas cancerous ovaries had a higher Fp redox ratio. ${ }^{42}$ Our results are consistent with this study. With the transgenic mouse model, we previously also saw a higher Fp redox ratio in premalignant pancreas. ${ }^{12}$ Results from cell culture studies show higher NADH but lower FAD in some BC lines than in the normal cell line MCF10A, resulting in a significantly higher $\mathrm{NADH} / \mathrm{FAD}$ ratio (lower Fp redox ratio) in the $\mathrm{BC}$ lines. ${ }^{38}$ However, the apparently inconsistent results may be due to the significant difference in cellular microenvironment between cell cultures and tumor tissues. 
Usually breast tumor tissue has higher cell density than normal breast tissue. ${ }^{48}$ This might partially explain our observed higher Fp and NADH in cancerous tissue but cannot explain the higher Fp redox ratio, which is largely independent of cell density. The higher Fp redox ratio in cancerous tissues may indicate a fundamental difference of the mitochondrial metabolic function and redox state from that of normal tissues.

Chance and Williams ${ }^{49,50}$ previously defined five distinct redox states of the mitochondria based on the isolated mitochondria with various levels of ADP, substrates, and oxygen supply. When oxygen level is zero, the mitochondria are in state 5 and their respiration rate reaches zero. Under such a state, NADH is high but Fp is low. The tissue aliquots collected for our study were on an average of $25 \mathrm{~min}$ after the breasts or breast tissue chunks had been removed from the body and sitting at room temperature. Therefore, it is possible their mitochondria were shifting toward state 5 due to low oxygen. It is known that for normal tissues, respiring activity is inhibited under hypoxia, resulting in higher NADH and lower Fp signals. However, tumor tissues, even under hypoxia, may still have high respiring activity. ${ }^{51,52}$ We do not know if this is the case for breast tumors. Assuming that breast tumor cells behave the same as normal cells and tumor tissues were more hypoxic than normal tissues, one should expect a decrease in Fp associated with an increase of NADH in tumors compared to normal tissues. However, this is not consistent with our results. It is possible that there was actually a large percentage of decrease in Fp in tumor tissue compared to normal tissue when shifting toward more hypoxic state. However, due to higher cell density in tumor tissue, total Fp content in tumor tissue remained higher than that in normal tissue. To better understand the results reported here, we need to obtain a more quantitative and complete picture about tissue biology, biochemistry and metabolism, and their intratumor heterogeneities.

An age-related NADH content decrease of BC patients with age 55 or older was reported in both tumor and normal tissues. ${ }^{33}$ Another study reported that both $\mathrm{NADH}$ and FAD signal decreased with increasing patient age for normal cervical tissue and the premenopausal patients $(<41 \mathrm{yr})$ had relatively higher $\mathrm{NADH}$ and FAD than the postmenopausal ones $(>41 \mathrm{yr}){ }^{53}$ We did not find any age dependence of the redox indices in this study for both tumor and normal tissues. More patients should be included to confirm our result.

The autofluorescence decay after tissue resection but before being frozen has been a concern for optical studies. ${ }^{36,54-56}$ The resected breasts/tissue chunks from which the biopsies were collected had been kept at room temperature for an average of 25 min. Therefore, it is likely that both NADH and Fp contents in these tissues had changed during this time period before collecting tissue aliquots for the redox scanning. The observed redox ratio only represented the metabolic state of the tissue aliquots at the time of snap-freezing. How well these redox measurements provide clinically useful information still needs to be further investigated.

The present study can be improved in the following aspects in the future. First, due to large interpatient variations in the redox indices, more patients should be included with a more standardized protocol on collecting tissue aliquots in order to evaluate the redox discriminating power more accurately. Second, by collecting larger size tissues, we should verify that the control specimens are truly noncancerous by histology. Third, by performing tissue heterogeneity analysis, we may generate more information that could improve cancer detection. This is supported by our previous works, where Gaussian curve fitting technique helped to distinguish precancer from normal ${ }^{12}$ and p53 status of colon cancer in mouse models. ${ }^{11}$ Fourth, we can include premalignant breast biopsies to study the redox difference among normal breast, atypia, and breast carcinomas. Lastly, as our results suggest the value of the redox indices for cancer management, we need to develop noninvasive imaging methods for monitoring the redox state in vivo.

\section{Conclusions}

This study highlights the differences in the mitochondrial redox state between cancerous and normal tissues via optically imaging biopsy specimens from BC patients. We found that the redox indices, such as Fp or NADH or the Fp redox ratio, alone could potentially be used as a discriminating index for $\mathrm{BC}$ diagnosis.

\section{Disclosures}

No conflicts of interest, financial or otherwise, are declared by the authors.

\section{Acknowledgments}

This work was supported by Susan G. Komen Foundation Grant No. KG081069 (L. Z. Li), the National Institutes of Health (NIH) Grant Nos. R01CA155348 and R01CA191207 (L. Z. Li), the National Cancer Institute (NCI) Cancer Center Support Grant No. 2-P30-CA-016520-35 (J. Tchou), the Linda and Paul Richardson Breast Cancer Research Funds (J. Tchou), Komen Leadership Grant (J. Tchou), the Center of Magnetic Resonance and Optical Imaging-an NIH supported research resource No. P41EB015893 (R. Reddy). We would like to acknowledge the assistance from Ms. Lily Moon during the initial collection and handling of the clinical biopsy samples. We would like to thank Professor Paul. J. Zhang (a certified pathologist) for his advice on this study.

\section{References}

1. R. A. Hubbard et al., "Cumulative probability of false-positive recall or biopsy recommendation after 10 years of screening mammography: a cohort study," Ann. Intern. Med. 155(8), 481-492 (2011).

2. L. E. Pace and N. L. Keating, "A systematic assessment of benefits and risks to guide breast cancer screening decisions," J. Am. Med. Assoc. 311(13), 1327-1335 (2014).

3. R. J. Brenner et al., "Stereotactic core-needle breast biopsy: a multiinstitutional prospective trial," Radiol. 218(3), 866-872 (2001).

4. S. H. Parker et al., "Percutaneous large-core breast biopsy: a multiinstitutional study," Radiol. 193(2), 359-364 (1994).

5. H. N. Xu and L. Z. Li, "Quantitative redox imaging biomarkers for studying tissue metabolic state and its heterogeneity," J. Innovative Opt. Health Sci. 7(2), 1430002 (2014).

6. H. N. Xu et al., "Quantitative mitochondrial redox imaging of breast cancer metastatic potential," J. Biomed. Opt. 15(3), 036010 (2010).

7. K. Cai et al., "Breast cancer redox heterogeneity detectable with chemical exchange saturation transfer (CEST) MRI," Mol. Imaging Biol. 16(5), 670-679 (2014).

8. H. N. Xu et al., "Characterizing the metabolic heterogeneity in human breast cancer xenografts by 3D high resolution fluorescence imaging," SpringerPlus 2(1), 73 (2013).

9. H. N. Xu et al., "Is higher lactate an indicator of tumor metastatic risk? A pilot MRS study using hyperpolarized 13C-pyruvate," Acad. Radiol. 21(2), 223-231 (2014).

10. H. N. Xu, J. Tchou, and L. Z. Li, "Redox imaging of human breast cancer core biopsies: a preliminary investigation," Acad. Radiol. 20(6), 764-768 (2013). 
11. H. N. Xu et al., "Imaging the redox states of human breast cancer core biopsies," Adv. Exp. Med. Biol. 765, 343-349 (2013).

12. H. N. Xu, S. Nioka, and L. Z. Li, "Imaging heterogeneity in the mitochondrial redox state of premalignant pancreas in the pancreas-specific PTEN-null transgenic mouse model," Biomarker Res. 1, 6 (2013).

13. H. N. Xu et al., "Redox imaging of the p53-dependent mitochondrial redox state in colon cancer ex vivo," J. Innovative Opt. Health Sci. 6, 1350016 (2013).

14. L. Z. Li et al., "Quantitative magnetic resonance and optical imaging biomarkers of melanoma metastatic potential," Proc. Natl. Acad. Sci. U. S. A. 106(16), 6608-6613 (2009).

15. L. Z. Li et al., "Predicting melanoma metastatic potential by optical and magnetic resonance imaging," Adv. Exp. Med. Biol. 599, 67-78 (2007).

16. R. A. Cairns, I. S. Harris, and T. W. Mak, "Regulation of cancer cell metabolism," Nat. Rev. Cancer 11(2), 85-95 (2011).

17. R. Banerjee, Redox Biochemistry, John Wiley \& Sons, Hoboken, New Jersey (2008).

18. W. H. Ying, "NAD" $/ \mathrm{NADH}$ and $\mathrm{NADP}^{+} / \mathrm{NADPH}$ in cellular functions and cell death: regulation and biological consequences," Antioxid. Redox Signaling 10(2), 179-206 (2008).

19. L. Z. Li, "Imaging mitochondrial redox potential and its possible link to tumor metastatic potential," J. Bioenerg. Biomembr. 44, 645-653 (2012).

20. A. Chiarugi et al., "The NAD metabolome-a key determinant of cancer cell biology," Nat. Rev. Cancer 12(11), 741-752 (2012).

21. R. B. Hamanaka and N. S. Chandel, "Warburg effect and redox balance," Science 334(6060), 1219-1220 (2011).

22. B. Chance et al., "Oxidation-reduction ratio studies of mitochondria in freeze-trapped samples. NADH and flavoprotein fluorescence signals," J. Biol. Chem. 254(11), 4764-4771 (1979).

23. L. Z. Li et al., "Mitochondrial redox imaging for cancer diagnostic and therapeutic studies," J. Innovative Opt. Health Sci. 2, 325-341 (2009).

24. B. Quistorff, J. C. Haselgrove, and B. Chance, "High spatial resolution readout of 3-D metabolic organ structure: an automated, low-temperature redox ratio-scanning instrument," Anal. Biochem. 148(2), 389-400 (1985).

25. I. Georgakoudi and K. P. Quinn, "Optical imaging using endogenous contrast to assess metabolic state," Ann. Rev. Biomed. Eng. 14, 351-367 (2012).

26. B. Chance et al., "Intracellular oxidation-reduction states in vivo," Science 137, 499-508 (1962).

27. K. Ozawa et al., "Linear correlation between acetoacetate/beta-hydroxybutyrate in arterial blood and oxidized flavoprotein/reduced pyridine nucleotide in freeze-trapped human liver tissue," Biochim. Biophys. Acta 1138(4), 350-352 (1992).

28. K. P. Quinn et al., "Quantitative metabolic imaging using endogenous fluorescence to detect stem cell differentiation," Sci. Rep. 3, 3432 (2013).

29. A. Varone et al., "Endogenous two-photon fluorescence imaging elucidates metabolic changes related to enhanced glycolysis and glutamine consumption in precancerous epithelial tissues," Cancer Res. 74(11), 3067-3075 (2014).

30. R. R. Alfano et al., "Fluorescence spectra from cancerous and normal human breast and lung tissues," IEEE J. Quantum Electron. 23(10), 1806-1811 (1987).

31. R. R. Alfano et al., "Optical spectroscopic diagnosis of cancer and normal breast tissues," J. Opt. Soc. Am. B 6(5), 1015-1023 (1989).

32. R. C. Benson et al., "Cellular autofluorescence-is it due to flavins?" J. Histochem. Cytochem. 27(1), 44-48 (1979).

33. A. Uppal and P. K. Gupta, "Measurement of NADH concentration in normal and malignant human tissues from breast and oral cavity," Biotechnol. Appl. Biochem. 37(Pt. 1), 45-50 (2003).

34. S. K. Majumdar and P. K. Gupta, "Synchronous luminescence spectroscopy of human breast tissues," Proc. SPIE 3252, 169-178 (1998).

35. P. K. Gupta, S. K. Majumder, and A. Uppal, "Breast cancer diagnosis using N2 laser excited autofluorescence spectroscopy," Lasers Surg. Med. 21(5), 417-422 (1997).

36. J. A. Delgado et al., "EX-vivo autofluorescence measurements of human tissues," in AIP Conf. Proc., Vol. 682, No. 1, pp. 30-37 (2003).

37. M. V. Chowdary et al., "Autofluorescence of breast tissues: evaluation of discriminating algorithms for diagnosis of normal, benign, and malignant conditions," Photomed. Laser Surg. 27(2), 241-252 (2009).

38. L. Z. Li and N. Sun, "Autofluorescence perspective of cancer diagnostics," in Natural Biomarkers for Cellular Metabolism, V. V. Ghukasyan and A. A. Heikal, Eds., pp. 273-298, CRC Press, Boca Raton, Florida (2014).

39. H. N. Xu et al., "Heterogeneity of mitochondrial redox state in premalignant pancreas in a PTEN null transgenic mouse model," Adv. Exp. Med. Biol. 701, 207-213 (2011).

40. N. Ramanujam et al., "Low temperature fluorescence imaging of freeze-trapped human cervical tissues," Opt. Express 8(6), 335-343 (2001).

41. A. T. Shah and M. C. Skala, "EX vivo label-free microscopy of head and neck cancer patient tissues," Proc. SPIE 9329, 93292B (2015).

42. N. D. Kirkpatrick, M. A. Brewer, and U. Utzinger, "Endogenous optical biomarkers of ovarian cancer evaluated with multiphoton microscopy," Cancer Epidemiol. Biomarkers Prev. 16(10), 2048-2057 (2007).

43. H. N. Xu et al., "Differentiating cancerous from normal breast tissue by redox imaging," Proc. SPIE 9303, 93032R (2015).

44. H. N. Xu et al., "Quantitative redox scanning of tissue samples using a calibration procedure," J. Innovative Opt. Health Sci. 2, 375-385 (2009).

45. H. N. Xu et al., "Calibration of redox scanning for tissue samples," Proc. SPIE 7174, 71742F (2009).

46. M. W. Conklin et al., "Fluorescence lifetime imaging of endogenous fluorophores in histopathology sections reveals differences between normal and tumor epithelium in carcinoma in situ of the breast," Cell Biochem. Biophys. 53(3), 145-157 (2009).

47. S. K. Majumder, N. Ghosh, and P. K. Gupta, "N2 laser excited autofluorescence spectroscopy of formalin-fixed human breast tissue," J. Photochem. Photobiol. B 81(1), 33-42 (2005).

48. H. J. Bloom and W. W. Richardson, "Histological grading and prognosis in breast cancer; a study of 1409 cases of which 359 have been followed for 15 years," Br. J. Cancer 11(3), 359-377 (1957).

49. B. Chance and G. R. Williams, "A method for the localization of sites for oxidative phosphorylation," Nature 176(4475), 250-254 (1955).

50. B. Chance and G. R. Williams, "Respiratory enzymes in oxidative phosphorylation. III. The steady state," J. Biol. Chem. 217, 409-427 (1955).

51. A. Le et al., "Tumorigenicity of hypoxic respiring cancer cells revealed by a hypoxia-cell cycle dual reporter," Proc. Natl. Acad. Sci. U. S. A. 111(34), 12486-12491 (2014).

52. A. Roesch et al., "Overcoming intrinsic multidrug resistance in melanoma by blocking the mitochondrial respiratory chain of slow-cycling JARID1B(high) cells," Cancer Cell 23(6), 811-825 (2013).

53. C. K. Brookner et al., "Autofluorescence patterns in short-term cultures of normal cervical tissue," Photochem. Photobiol. 71(6), 730-736 (2000).

54. R. Cicchi et al., "Time- and spectral-resolved two-photon imaging of healthy bladder mucosa and carcinoma in situ," Opt. Express 18(4), 3840-3849 (2010).

55. K. T. Schomacker et al., "Ultraviolet laser-induced fluorescence of colonic tissue: basic biology and diagnostic potential," Lasers Surg. Med. 12(1), 63-78 (1992).

56. G. M. Palmer et al., "Optimal methods for fluorescence and diffuse reflectance measurements of tissue biopsy samples," Lasers Surg. Med. 30(3), 191-200 (2002).

He N. Xu, MS in optics, MS and PhD in chemistry, is a senior research investigator of radiology at the University of Pennsylvania. She joined Dr. Lin Li lab in 2006 and also worked closely with Dr. Britton Chance for over 3 years to optically investigate the roles of redox state in normal and diseased tissues. Her current research focuses on the development and clinical translation of optical imaging techniques to study tumor progression.

Julia Tchou, MD, PhD, is a breast cancer surgeon and a physicianscientist whose focus is to deliver the best of care to breast cancer patients. Her overall goal is to lessen breast cancer burden by combining translational research and delivering outstanding clinical care to women diagnosed with breast cancer. Her research is focused on the development of targeted therapy for invasive breast carcinoma. 
Min Feng received her bachelor's degree in biochemistry from Ursinus College in 2015. Currently, she is working as a research technician under the supervision of Dr. He N. Xu. Her research focuses on performing redox imaging of tumors and cell cultures and the related data processing.

Huaqing Zhao is an assistant professor of biostatistics and epidemiology at the Temple University School of Medicine. He graduated with a master in biostatistics and $\mathrm{PhD}$ in epidemiology from the University of Pennsylvania. He worked as a biostatistician for over 20 years in the Children's Hospital of Philadelphia's and joined Temple University in January 2013. He conducted collaborative research in a variety of areas including oncology, pulmonary, neurology, AIDS research, endocrinology, cardiology, radiology, and immunology.

Lin Z. Li, MS in physics in 1994, PhD in molecular biophysics in 2000, is an associate professor of radiology and directs the Britton Chance Laboratory of Redox Imaging at the University of Pennsylvania. Prior to 2004 , he developed original NMR methods that contributed fundamentally to the development of quantitative susceptibility mapping. Since 2004, he has been employing and developing NMR/optical imaging methods to interrogate cellular metabolism and redox state during tumor progression to invasion/metastasis. 